\title{
Availability of orthodontic smartphone apps
}

\author{
Siddiqui NR ${ }^{1}$, Hodges $\mathrm{SJ}^{2}$, Sharif $\mathrm{MO}^{3}$
}

1. Miss Nausheen Siddiqui

Specialty Registrar in Orthodontics, University College London Hospitals Foundation Trust, Eastman Dental Hospital

2. Miss Samantha Hodges

Consultant in Orthodontics, University College London Hospitals Foundation Trust, Eastman Dental Hospital

3. Mr Mohammad Owaise Sharif

Clinical Lecturer/Honorary Consultant in Orthodontics, University College London Eastman Dental Institute 


\section{ABSTRACT}

\section{Objective}

To identify the availability and to profile orthodontic smartphone apps published on the UK Apple App Store and Google Play Store.

\section{Design}

A review of available smartphone apps.

\section{Setting}

London, U.K.

\section{Methods}

The Apple App Store and Google Play Store were accessed in October 2018; all orthodontic apps with a title and description available in English were included. Each app was classified according to the following criteria: name; target audience (patient or clinician); focus; platform (Apple App Store and/or Google Play Store); cost; rating and number of ratings; size; creation date; country of development and developer.

\section{Results}

A total of 305 orthodontic related apps were identified for inclusion. The majority of apps were aimed at patients $(n=241)$ and the most of these were focussed on gaming $(n=136)$, many apps were also found to be developed by specialist practices $(n=63)$ and to provide knowledge for patients $(\mathrm{n}=56)$. Two hundred and ninety-five apps were free, with some of these including the option of paying for in-app purchases. Most apps were developed in the USA $(n=84)$.

\section{Conclusions}


There has been an increase in the availability of orthodontic apps in recent years. The majority of available apps are patient-focused, most commonly games. A number of apps aim to elicit a behaviour change in our patients; therefore, there is a need to assess the quality and educational content of these.

\section{Keywords}

App, mobile phone, smartphone, orthodontics.

\section{INTRODUCTION}

A recent study at the University College London Hospitals NHS Foundation Trust Eastman Dental Hospital assessed the utilisation of mobile phones by 100 consecutive orthodontic patients. The study concluded that $90 \%$ of patients had access to a smartphone and that all patients accessed either the Apple App Store or the Google Play Store to obtain apps (Sharif et al., 2019). The availability of apps is increasing; a recent report concluded that the Google Play Store is the largest app store $(3,800,000$ apps $)$, followed by the Apple App Store $(\mid 2,000,000$ apps) (Statista, 2018a).

In orthodontics, smartphone applications can be utilized to provide personalised treatment information such as treatment progress photographs and reminders to wear elastics, aligners or attend appointments. The current evidence suggests that apps utilised to support orthodontic treatment are effective in improving appointment attendance, reducing appliance breakages ( $\mathrm{Li}$ et al., 2016), improving oral health and minimising the risks of treatment (Alkhadi et al., 2017, Zotti et al., 2015). For the clinician, apps have the capability of aiding training (including the provision of revision aids), providing clinical support (mentoring and 
peer review) and assisting with treatment planning.

In 2013, it was reported that 19 orthodontic apps were available on the Apple App Store and Google Play Store (Singh, 2013). Eight of these apps were patient-focused and 11 apps were clinician-focused. In 2014, this number had increased to 119 (Baheti and Toshniwal, 2014), 23 of these were patient-focused; 27 were clinician-focused; 53 were related to marketing of specialist practice; and the remaining 16 were games. The most recent evaluation of app availability revealed the number of orthodontic apps had increased still further to 354 (Gupta and Vaid, 2017), with 292 of these aimed at clinicians and 62 at patients.

To date there has not been a comprehensive review of available orthodontic apps with regards to their ratings/ reviews, cost and size. App cost is a significant factor influencing app utilisation with a large proportion of patients reporting that they would not be willing to pay for a healthcare app (Krebs and Duncan, 2015). App reviews are also important; it has been shown that consumer reviews correlate highly with download counts and are a key measure of an app's success (Khalid et al., 2014). Furthermore, app size is also important to consider; if the app is too large it may be difficult or expensive for users to download (Boshell, 2017).

The primary aim of this study was to identify the availabilityof orthodontic smartphone apps found in the Apple App Store and Google Play Store in the UK. The secondary aim was to analyse the apps with regards to a number of features including the target audience, focus of the app, cost, rating and number of ratings, size, year of publication, country of development and developer. 


\section{MATERIALS AND METHODS}

The Apple App Store and Google Play Store were accessed in October 2018 (London, UK) utilising the following mobile phones and operating systems:

- Apple iPhone X using iOS 12

- $\quad$ Vodafone Smart N9 Lite using Android 8.1

The following key words were used to identify apps for inclusion: 'braces', 'orthodontist', 'orthodontics', 'aligner', 'fixed appliance', 'functional appliance', 'removable appliance', 'retainer', 'Incognito' and 'Invisalign'. These were identified as being popular search words related to orthodontics using 'Google Adwords'.

The following inclusion criteria were used to identify apps for inclusion:

- Orthodontic apps (general dentistry apps with an orthodontic element were excluded).

- Apps available on the UK Apple App Store and Google Play Store.

- App in English only.

\section{Data collection:}

The following information was entered into a Microsoft Excel spreadsheet for all included apps: 
- Name

- Country of development

- Cost (basic +/- upgrade cost)

- Creation date

- Developer

- Focus of app (i.e. game, marketing)

- Platform (Apple App Store and/or Google Play Store)

- Rating and number of ratings

- Size

- Target audience (patient or clinician)

- Year of development

Before data collection the authors developed and defined categories relating to app focus (Table 1) and 10 apps were piloted independently to assess the robustness of the criteria. It was intended that if any further categories arose during data collection, these would be discussed by the research team and if appropriate added.

All apps were assessed and categorised relating to their focus by two independent assessors (NRS and MOS). It was intended that any disagreements would be resolved by discussion; where this was not possible, a third researcher (SJH) would be consulted to mediate and obtain consensus.

Where data were missing from any of the categories, e.g. file size or country of developer, attempts were made by NRS to obtain this by contacting the developer via email. 


\section{RESULTS}

The search of app stores retrieved 952 apps. After independent assessment by two researchers (NRS and MOS), 305 apps were identified for inclusion. There was 100\% agreement between the authors regarding app inclusion. A total of 140 apps were available on the Google Play Store only, 117 on the Apple App Store only and 48 were available on both. The majority of apps were aimed at patients $(n=241), 60$ apps were aimed at clinicians and the remaining four apps had both patient- and clinician-focused domains. The year of development is summarised in Figure 1 and the number of apps developed can be seen to be increasing year on year.

The most common focus of the apps retrieved was gaming $(\mathrm{n}=136)$, followed by apps developed by a specialist practice $(n=63)$, knowledge for patients $(n=56)$, marketing $(n=$ 32), behaviour change $(n=31)$, clinician revision and knowledge $(n=14)$, treatment planning aids $(n=13)$, conference apps $(n=10)$, clinician support with treatment $(n=9)$, screening patients for treatment $(n=6)$, journals $(n=4)$, professional societies $(n=4)$, practice building $(n=3)$, clinician portfolio $(n=2)$, laboratory $(n=2)$, patient treatment tracker for clinicians $(n$ $=2)$ and remote orthodontist $(n=1)$. Several apps were relevant to more than one category. The focus of the apps was assessed independently by two assessors (NRS and MOS); there was agreement for 295 apps $(96.7 \%)$. The focus of the remaining 10 apps was agreed after discussion.

The majority of apps were free $(n=272), 10$ required payment for access and 23 apps were free but contained in-app purchase options. The cost of apps was in the range of $£ 0.99-£ 28.99$ and further details are summarised in Table 2. In-app purchase costs were in the range of $£ 0.99$ $£ 229.99$. Most in-app purchases were under $£ 2$ and allowed for adverts to be removed. Five 
apps had in-app purchases over $£ 20$ and these were all aimed at clinicians; to buy online learning material, treatment aids or journal subscriptions.

App ratings are displayed as a score of $1-5$ on the app store where 1 is the poorest score and 5 is the best score. The majority of apps did not have a sufficient number of ratings to display a score, however, of those that did, $88 \%$ scored a minimum of 3 out of 5 (Figure 2). The number of app ratings is a further indicator of app popularity (Figure 3).

A large variation of app size was noted (range $<1-236.7 \mathrm{MB}$ ); however, the majority of apps ( $\mathrm{n}=256)$ were $<40 \mathrm{MB}$. Interestingly, no obvious correlation was found between the size and focus of the apps; the apps $>100 \mathrm{MB}(\mathrm{n}=7)$ were a mixture of patient- and clinician targeted apps with different focuses.

The data for the country of app development was available from 172 apps. Developers were contacted to obtain missing data; however, to date no responses have been received in relation to the remaining 133 apps. The country of app development varied $(n=23)$ and these data are summarised in Table 3 which shows the top six developing countries where 141 of the apps were developed. Thirty-eight developers created more than one of the included apps which accounted for 108 of the 305 apps analysed.

\section{DISCUSSION}

The number of available orthodontic apps is increasing year on year. The number of apps developed in 2018 does not seem to follow this trend, however, it must be noted that data was collected in October 2018 and it may be assumed that further apps were published after this 
timepoint in 2018. This study identified that 305 orthodontic apps were available on the UK Apple App Store and Google Play Store; this is a significant increase from the only other UKbased orthodontic app study that identified 19 apps in 2013 (Singh, 2013).

Gupta and Vaid (2017) identified more apps $(\mathrm{n}=354)$ than this current study; however, there could be a number of reasons to account for this. First, the methodology and inclusion criteria were different. Second, app availability is fluid in that it can reduce and increase at any time because app stores are updated and apps become non-functional or developers remove apps. Third, the Gupta and Vaid (2017) study was carried out in India where the availability of apps on the app stores may be different.

The focus of the largest number of apps identified in this study related to gaming (patientfocused) and not orthodontic treatment or clinician support. This finding correlates to the high proportion of games across all app fields; games account for $25 \%$ of app volume on the Apple App Store (Statista, 2018b). The most popular type of game identified in this study involved placing stickers on a selfie to see what the patient would look like with fixed appliances. Other games included changing module colours, designing a brace and creating a video using a series of selfies to monitor treatment progress. The large volume of games highlights the demand by orthodontic patients while incorporating the trend for social media, particularly selfies.

Other findings regarding the focus of the apps were:

- Apps developed by specialist practice (mostly developed in the USA, $n=52$ ) were aimed at primarily engaging patients in treatment. Many of these apps also provided knowledge for patients (including pretreatment information and in-treatment information, e.g. management of emergencies), behaviour change techniques 
(coordinated sets of activities designed to change specified behaviour patterns (Michie et al., 2011)), games and marketing.

- Another popular focus of orthodontic apps was to elicit a behaviour change. Behaviour change techniques found in these apps most commonly included: reminders to wear elastics; aligners or attend appointments; instructions on how to perform a behaviour (e.g. oral hygiene instruction); self-monitoring of behaviour (i.e. compliance tracker); and rewards for complying with treatment.

- Clinician revision and/or knowledge-focused apps included blogs, glossaries, revision aids and seminars contributing to core professional development.

- Treatment planning apps mostly consisted of cephalometric and space analyses.

The majority of apps were free. This is advantageous as Krebs and Duncan (2015) identified cost to be a significant barrier to downloading healthcare apps among respondents, with a large proportion indicating that they would not pay for a healthcare app. Murfin (2013) also showed that apps are primarily chosen according to price with users frequently preferring free apps to those which charge, especially when there may be a similar free alternative, despite the ratings/reviews. It was reported, in 2018 , that $94 \%$ of all apps available on the Google Play Store were free (Statista, 2018c); this finding is consistent with this study as 97\% of apps were free of charge. However, paid apps may have advantages, for example there may be a higher perceived value to the consumer and therefore they may be more likely to be loyal to apps which they pay for. The more expensive apps were clinician-focused.

Most of the apps identified (84\%) were < 40 MB. In 2017, it was reported that the average file size was $38 \mathrm{MB}$ for Apple apps and $15 \mathrm{MB}$ for Android apps (Boshell, 2017). No obvious correlation was found between app size and any of the other categories, such as target audience, 
focus, rating, number of ratings, etc. The literature has, however, reported significant differences in Apple file size between categories, with apps containing games being the largest (Boshell, 2017).

At present, the majority of orthodontic apps on the Apple App Store $(n=165)$ do not have a rating, this is due to an insufficient number of user ratings. This lack of data means it is not possible to use this measure as a reliable indicator of app popularity. Evidence has shown that consumer reviews, which can be extrapolated to ratings, correlate highly with download counts and are a key measure of an app's success (Khalid et al., 2014). Patients are more likely to download rated apps due to their perceived credibility by other app users. The average rating of the 48 orthodontic apps that were available in both app stores was $4.6 / 5$, which was higher than the average rating of all orthodontic apps (3.8/5). It is possible that the more popular apps are duplicated across both platforms so that they are accessible to more users. Interestingly, apps with the highest number of ratings were all patient-focused games.

This study recorded app developer details for two main purposes: firstly, to assess the credibility of the source; and, secondly, to assess if developers created multiple apps. It was found that very few developers were recognised credible sources. None of the developers that created multiple apps appeared to be orthodontists or professional societies. It is also unclear whether app content was overseen by an orthodontic clinician; therefore, the quality of the content may be questionable.

\section{Limitations of research}


There were incomplete data available on the app stores for some of the categories including ratings, number of ratings, country of development and app size. The rapid proliferation of apps and fluidity of the apps available on these app stores means that the results are most accurate at the time of data collection. Singh (2013), Baheti and Toshniwal (2014) and Gupta and Vaid (2017) previously assessed the availability of orthodontic apps. The analyses in these papers were mostly restricted to the app target audience and focus, with some analysis of cost and ratings. It is therefore difficult to further analyse changes in app demographics in relation to these earlier publications. Furthermore, there were incomplete data available on the app stores for some of the categories including ratings, number of ratings, country of development and app size.

\section{Implications for patient care}

The majority of apps identified were games and did not aim to educate or modify patients' behaviour. However, a large number of apps were aimed at educating patients and modifying patients' behaviour, e.g. to improve compliance. It is likely that patients may use the information provided in these apps to help make decisions about their treatment. Therefore, the content and quality of these apps must be assessed prior to any recommendation in relation to their appropriateness for use in patient care.

The rapid proliferation of apps makes it is increasingly difficult for patients and healthcare professionals to identify high-quality apps (Cummings et al., 2013). The General Dental Council (GDC) provides Standards for the Dental Team and guidance on advertising (General Dental Council, 2013), which requires all material made public by members of the dental team to be truthful and credible. Selecting apps based on ratings and reviews does not necessarily 
correlate to the quality of the app (Girardello and Michahelles, 2010). Apps aiming to educate patients or modify behavior should contain evidence-based, peer-reviewed information, which is unlikely to be reflected by the star rating or reviews. Chen et al. (2015) evaluated the quality of the 800 most popular health and fitness smartphone apps and quantified their behaviour change techniques. They concluded that the most popular apps in terms of ratings scored below average on a number of quality features. The rating of an app should therefore be treated with caution and cannot be used reliably by patients or clinicians to determine its quality. The National Health Service (NHS) publishes a library of apps meeting NHS quality standards; however, no orthodontic apps have yet been listed. Importantly, to date there are no studies assessing the quality of these apps, the behaviour change techniques used and their knowledge content.

\section{CONCLUSION}

A total of 305 orthodontic-related apps were available on the UK Apple App Store and Google Play Store in October 2018. These were largely aimed at patients; the most common focuses areas were gaming, apps developed by specialist practices and those providing knowledge to patients. The majority of apps were free and the highest number of apps were developed in the USA. Apps can provide a number of benefits to clinicians and patients, which has been supported by several randomised controlled trials (Alkadhi et al., 2017; Li et al., 2016; Zotti et al., 2015); however, there is currently no formal published assessment of the orthodontic app quality, the behaviour change techniques utilised and their knowledge content. Patients may therefore be using apps to make treatment decisions by using information that may not be peerreviewed and evidence-based. Given the availability of apps targeted at orthodontic patients, 
there is a need to assess these apps and, if appropriate, navigate patients towards high-quality, effective apps.

\section{DECLARATION OF CONFLICTING INTEREST}

The authors declared no potential conflicts of interest with respect to the research, authorship and/or publication of this article

\section{FUNDING}

Mohammad Owaise Sharif was awarded the Royal College of Surgeons of England Faculty of Dental Surgery 70th Anniversary Research Fellowship. This funding has supported this project.

\section{APPENDICES}

\begin{tabular}{|c|c|c|}
\hline & Focus of app & Description \\
\hline 1 & Behaviour change & $\begin{array}{l}\text { Aims to improve patient compliance with treatment, e.g., reminders, instructions } \\
\text { on how to perform behaviour (i.e. elastic placement), rewards for compliance. }\end{array}$ \\
\hline 2 & Clinician portfolio & Clinician logs orthodontic cases. \\
\hline 3 & $\begin{array}{l}\text { Clinician revision and } \\
\text { knowledge }\end{array}$ & $\begin{array}{l}\text { Revision and/or education tools for clinicians including revision aids, continuing } \\
\text { professional development and seminars. }\end{array}$ \\
\hline 4 & $\begin{array}{l}\text { Clinician support with } \\
\text { treatment }\end{array}$ & Clinicians discuss cases or seek advice from other clinicians. \\
\hline 5 & Conferences & An app designed for a specific conference. \\
\hline 6 & Game & $\begin{array}{l}\text { Usually designed for patients and includes uploading selfies and adding stickers to } \\
\text { see what the patient looks like with braces on, changing module colours and } \\
\text { designing a brace. Orthodontic related games only, does not include generic games } \\
\text { on orthodontic apps. }\end{array}$ \\
\hline 7 & Journals & Access to a journal. \\
\hline 8 & Knowledge for patients & $\begin{array}{l}\text { Providing knowledge to patients including pre-treatment, mid-treatment and post- } \\
\text { treatment information about braces. }\end{array}$ \\
\hline 9 & Laboratories & An app designed by a laboratory for users of that laboratory. \\
\hline 10 & Marketing & $\begin{array}{l}\text { Promotion of products includes any app which specifically promotes a named } \\
\text { orthodontic brand. }\end{array}$ \\
\hline 11 & $\begin{array}{l}\text { Patient tracker for } \\
\text { clinicians }\end{array}$ & Clinician is able to monitor patients' progress or compliance with treatment. \\
\hline 12 & Specialist practice & $\begin{array}{l}\text { An app designed specifically by a specialist orthodontic practice for patients at the } \\
\text { practice. }\end{array}$ \\
\hline 13 & Practice building & Apps to improve efficiency or structure of a specialist practice. \\
\hline 14 & Remote orthodontist & $\begin{array}{l}\text { Allows remote access to an orthodontic clinician i.e. for advice for patient's in } \\
\text { treatment. Does not include sending pictures for screening for treatment. }\end{array}$ \\
\hline
\end{tabular}


15 Screening for treatment for patients

16 Professional societies

17 Treatment planning
Allows assessment of prospective orthodontic patients seeking an opinion on their suitability for treatment.

An app developed by a society for its' members.

Aids with treatment planning (diagnostic aids), e.g., cephalometric analyses and tooth-size analysis.

Table 1. Focus of apps with descriptions.

\begin{tabular}{|l|l|l|l|}
\hline App name & Basic cost & Focus & $\begin{array}{l}\text { Target } \\
\text { audience }\end{array}$ \\
\hline $\mathbf{1}$ & $£ 0.79$ & Game & Patient \\
\hline $\mathbf{2}$ & $£ 0.99$ & Game & Patient \\
\hline $\mathbf{3}$ & $£ 0.99$ & Game, knowledge & Patient \\
\hline $\mathbf{4}$ & $£ 0.99$ & Game & Patient \\
\hline $\mathbf{5}$ & $£ 1.99$ & Clinician portfolio & Clinician \\
\hline $\mathbf{6}$ & $£ 3.99$ & Clinician revision & Clinician \\
\hline $\mathbf{7}$ & $£ 4.49($ Google)/£7.99(Apple) & Clinician revision & Clinician \\
\hline $\mathbf{8}$ & $£ 8.99($ Google)/£9.99(Apple) & Clinician revision & Clinician \\
\hline $\mathbf{9}$ & $£ 23.64$ & Treatment planning & Clinician \\
\hline $\mathbf{1 0}$ & $£ 28.99$ & Treatment planning & Clinician \\
\hline
\end{tabular}

Table 2. Price and demographics of paid apps. 


\begin{tabular}{|l|l|}
\hline Country & Number of apps \\
\hline U.S.A. & 84 \\
\hline U.K. & 17 \\
\hline India & 15 \\
\hline Japan & 9 \\
\hline Australia & 8 \\
\hline Canada & 8 \\
\hline Other & 31 \\
\hline Unable to ascertain country & 133 \\
\hline
\end{tabular}

Table 3. Number of apps developed by the most popular countries. 


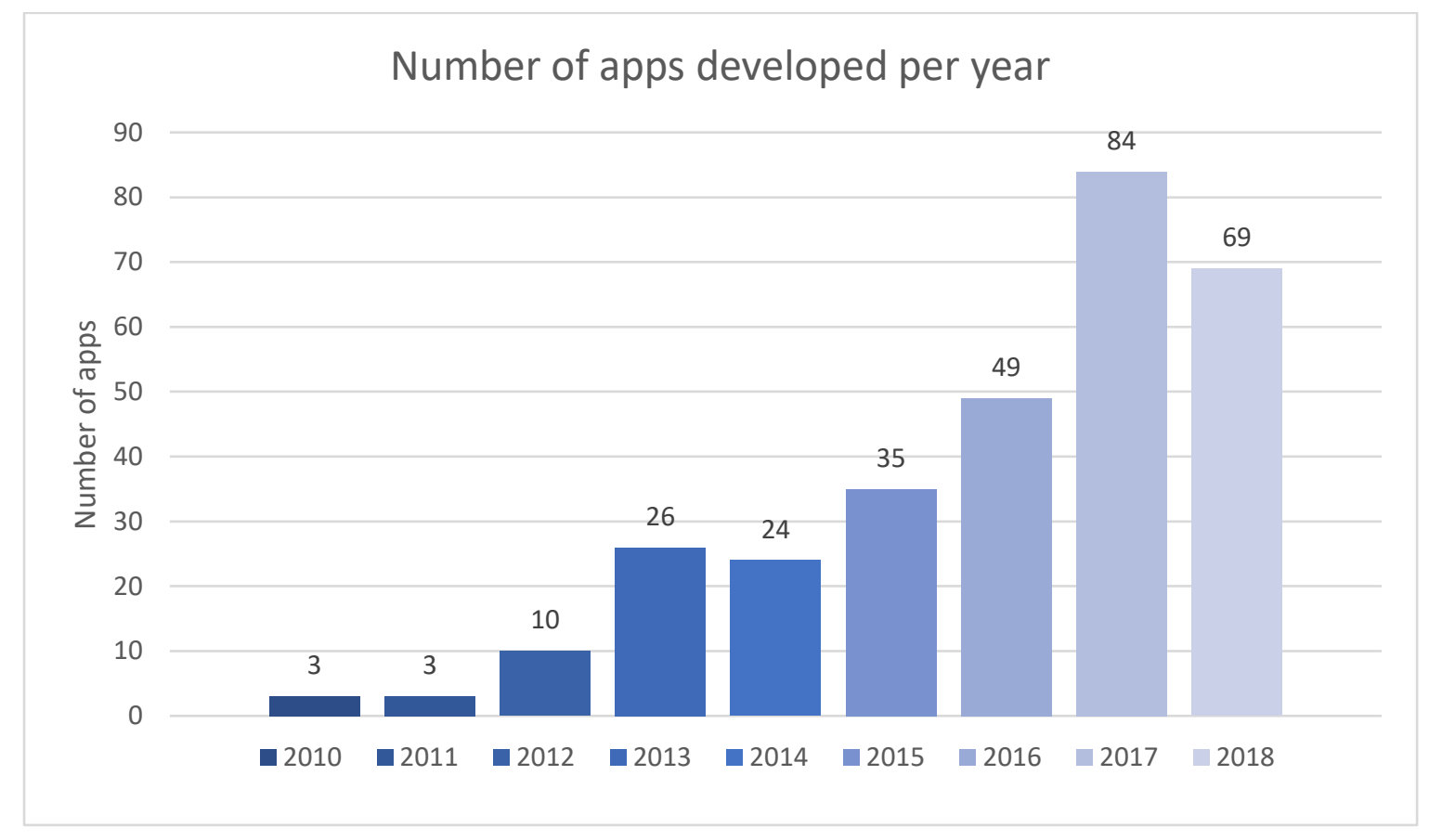

Figure 1. Number of apps developed per year. 


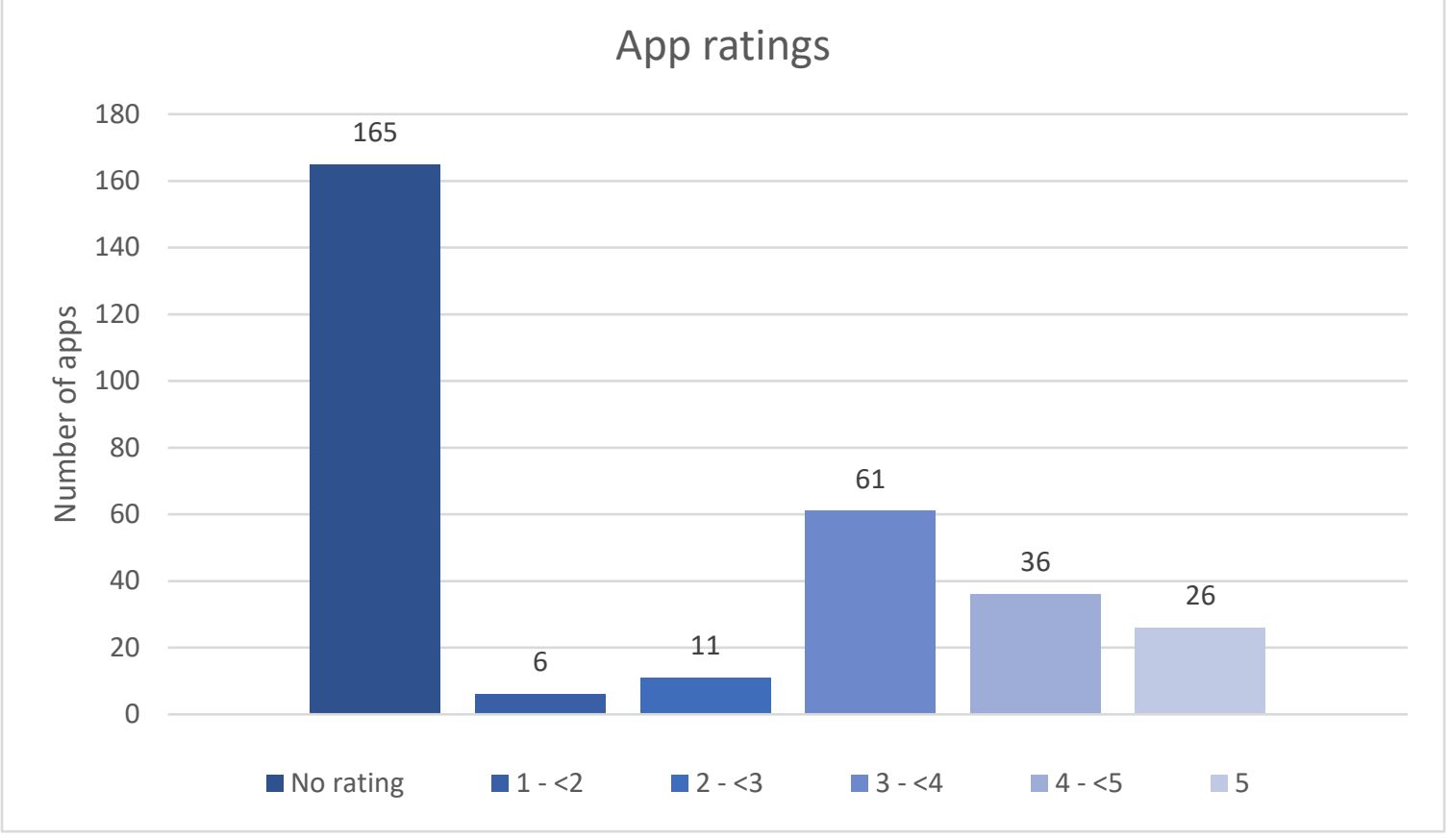

Figure 2. App ratings.

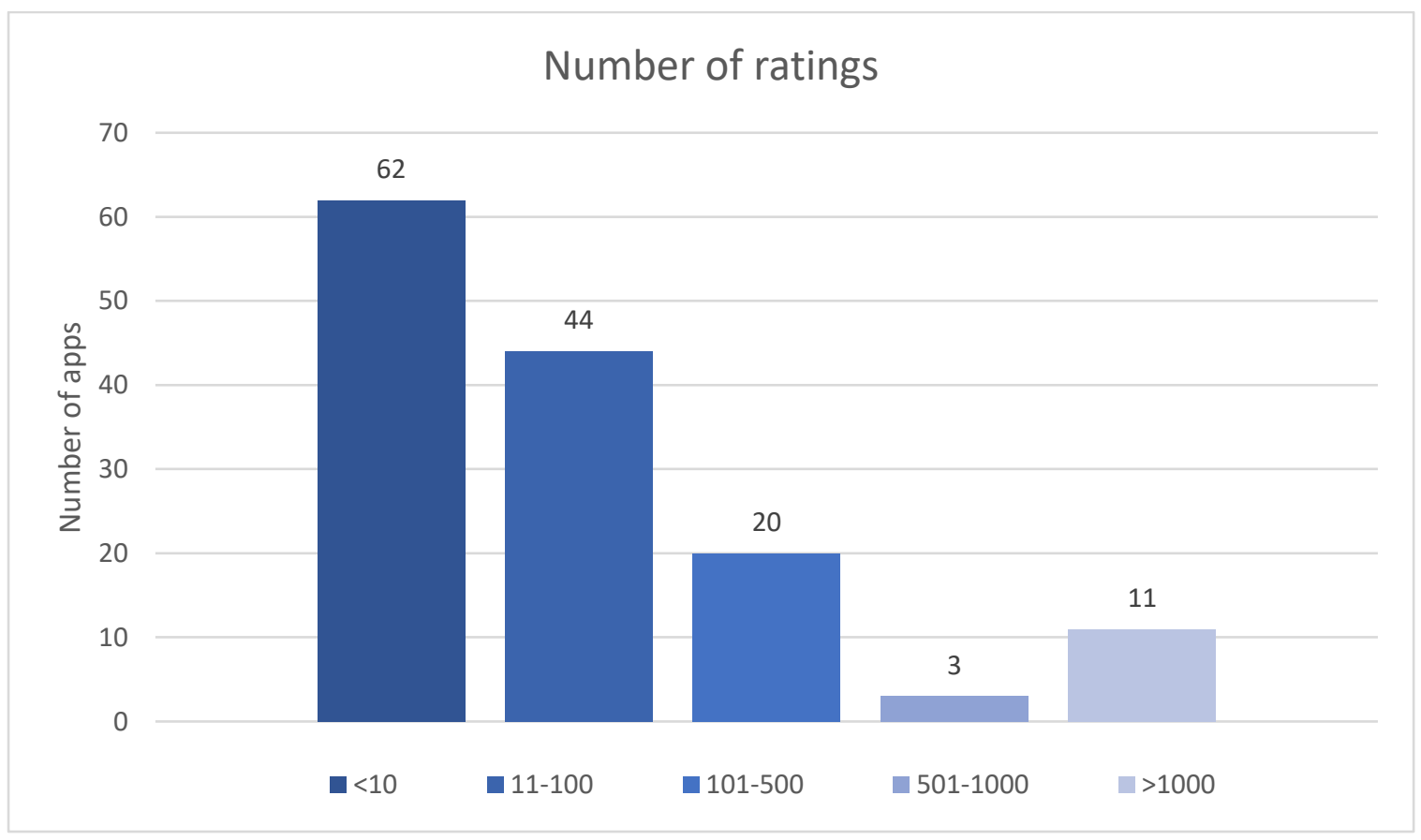

Figure 3. Number of ratings.

\section{REFERENCES}


Adoriasoft. 2017. Healthcare Mobile App Development and mHealth Apps in 2017. Las Vegas, NV: Adoriasoft. Available at: https://medium.com/@ Adoriasoft_Com/healthcaremobile-app-development-and-mhealth-apps-in-2017-eb307d4cad36. (accessed 1st March 2018).

Alkadhi OH, Zahid MN, Almanea RS, Althaqeb HK, Alharbi TH and Ajwa NM. 2017. The effect of using mobile applications for improving oral hygiene in patients with orthodontic fixed appliances: a randomised controlled trial. Journal of orthodontics. 44(3): 157-163.

Baheti MJ and Toshniwal N. 2014. Orthodontic apps at fingertips. Progress in orthodontics. 15(1): 36.

Boshell. 2017. Average app file size: data for android and iOS mobile apps. Available from: https://sweetpricing.com/blog/2017/02/average-app-file-size/. (accessed 2018 1st September).

Chen J, Cade JE and Allman-Farinelli M. 2015. The most popular smartphone apps for weight loss: a quality assessment. JMIR mHealth and uHealth. 3(4).

General Dental Council. 2013. Guidance on advertising.

Girardello A. and Michahelles F. 2010. AppAware: Which mobile applications are hot? Proceedings of the 12th international conference on Human computer interaction with mobile devices and services. 431-434

Gupta G and Vaid NR. 2017. The World of Orthodontic apps. APOS Trends in Orthodontics. 7(2): 73.

Khalid H, Shihab E, Nagappan M and Hassan AE. 2015. What do mobile app users complain about?. IEEE Software. 32(3): 70-77.

Knösel M, Jung K and Bleckmann A. 2011. YouTube, dentistry, and dental education. Journal of Dental Education. 75(12): 1558-1568.

Krebs P and Duncan DT. 2015. Health app use among US mobile phone owners: a national survey. JMIR mHealth and uHealth. 3(4).

Kreuter MW and Holt CL. 2001. How do people process health information? Applications in an age of individualized communication. Current Directions in Psychological Science. 10(6): 206-209.

Lena Y and Dindaroğlu F. 2017. Lingual orthodontic treatment: A YouTube ${ }^{\mathrm{TM}}$ video analysis. The Angle Orthodontist. 88(2): 208-214.

Li X, Xu ZR, Tang N, Ye C, Zhu XL, Zhou T and Zhao Z.H. 2016. Effect of intervention using a messaging app on compliance and duration of treatment in orthodontic patients. Clinical oral investigations. 20(8): 1849-1859.

Murfin M. 2013. Know your apps: an evidence-based approach to evaluation of mobile clinical applications. J Physician Assist Educ. 24(3): 38-40. 
Michie S, Van Stralen MM and West R. 2011. The behaviour change wheel: a new method for characterising and designing behaviour change interventions. Implementation science. $6(1): 42$.

Singh P. 2013. Orthodontic apps for smartphones. Journal of orthodontics. 40(3): 249-255.

Statista a. 2018. Number of smartphone users worldwide from 2014 to 2020 (in billions) Available from: https://www.statista.com/statistics/330695/number-of-smartphone-usersworldwide/. (accessed 1st March 2018)

Statista b. 2018. Most popular Apple App Store categories in September 2018, by share of available apps Available from: https://www.statista.com/statistics/270291/popular-categoriesin-the-app-store/. (accessed $27^{\text {th }}$ November 2018).

Statista c. 2018. Distribution of free and paid Andoid apps in the Google Play Store from $3^{\text {rd }}$ quarter 2017 to $1^{\text {st }}$ quarter 2018 Available from:

https://www.statista.com/statistics/266211/distribution-of-free-and-paid-android-apps/. (accessed 27 ${ }^{\text {th }}$ November 2018).

Sharif MO, Siddiqui NR, Hodges SJH. 2019. Patient awareness of apps in orthodontics. Journal of orthodontics. In Press- 1-5 DOI: 10.1177/1465312518821361

Kornstein S. 2015. The Rise of Mobile Phones: 20 Years of Global Adoption. Available from: https://blog.cartesian.com/the-rise-of-mobile-phones-20-years-of-global-adoption (accessed: $30^{\text {th }}$ January 2018)

Zotti F, Dalessandri D, Salgarello S, Piancino M, Bonetti S, Visconti L and Paganelli C. 2015. Usefulness of an app in improving oral hygiene compliance in adolescent orthodontic patients. The Angle Orthodontist. 86(1): 101-107. 